\title{
The Prognostic Value of the Derived Neutrophil-to-Lymphocyte Ratio in Transplantation-Ineligible Patients with Multiple Myeloma
}

\author{
Burak Uz \\ Department of Adult Hematology, Medicana International Samsun Hospital, Samsun, Turkey
}

Multiple myeloma (MM) is a clonal disorder characterized by the proliferation of plasma cells and their accumulation within the bone marrow. Due to its heterogenity and dynamic process, physicians are sometimes unable to predict the long-term prognosis of their patients in the pretreatment period. To solve this problem, some inflammatory-based new prognostic tools were introduced, like the neutrophil-to-lymphocyte ratio (NLR). The NLR, obtained from a routine complete blood count with a differential count, has been validated as a prognostic tool in solid tumors, and more recently in hematological malignancies including diffuse large B cell lymphoma (DLBCL), classical Hodgkin's lymphoma, MM, and peripheral T cell lymphoma [1]. The absolute lymphocyte count is not routinely documented in clinical trials and this is why the derived (d)NLR was developed by Proctor et al. [2]. The dNLR, simply calculated as absolute neutrophil count (ANC, cells/ $\mu \mathrm{L}$ ) divided by the difference between total leukocyte count and absolute neutrophil count (WBC ANC, cells $/ \mu \mathrm{L}$ ), can be determined from a complete blood count. It can be formulated as $\mathrm{dNLR}=\mathrm{ANC} /(\mathrm{WBC}-$ ANC). Proctor et al. [2] showed that dNLR was noninferior to NLR in a large cohort of patients with solid and hematological malignancies. However, unfortunately, hematologic disorders were not explicitly documented.

An Austrian study group was the first to evaluate the prognostic significance of baseline dNLR in 290 patients with DLBCL [3]. The 5-year overall survival (OS) for patients with a high dNLR $(\geq 4)$ was significantly better $(p<$ $0.043)$ than for those with a low dNLR $(<4)$. A high dNLR $(\geq 1.8)$ was also associated with shorter 5-year diseasefree survival (DFS) in DLBCL patients $(p<0.032)$. On multivariate analysis, older age ( $<60$ vs. $\geq 60$ years), advanced disease stage (stages I and II vs. stages III and IV), cell of origin as well as a high dNLR were identified as negative prognostic factors for both OS and progressionfree survival (PFS).

In the current issue of Acta Haematologica, a retrospective and multicenter study by Lee et al. [4] evaluated, for the first time, the prognostic value of dNLR in 176 symptomatic (active) and transplant-ineligible MM patients. All were newly diagnosed and had, at least, received the same protocol including bortezomib, melphalan, and prednisone (VMP). They were divided into 2 groups: low dNLR $(<1.51)$ and high dNLR $(\geq 1.51)$. The cut-off values were determined by receiver operating characteristics (ROC) curve analysis for the prediction of OS. The groups were similar in terms of age, sex, type of myeloma, stage (Durie-Salmon and International Staging System [ISS]), Eastern Cooperative Group performance status, serum $\beta_{2}$-microglobulin, albumin, creatinine, comorbidities, serum lactate dehydrogenase, and cumulative bortezomib dose. Only the number of skeletal lesions and cytogenetic abnormalities were significantly different in the 2 groups.

\section{KARGER}

(c) 2018 S. Karger AG, Basel 
OS was the primary end point of the study, while PFS, response rate, and safety were the secondary end points. At the end of the study, some important results were revealed. (a) The patients in the high dNLR group had a significantly worse 2-year OS rate than those in the low dNLR group ( 72.2 vs. $84.7 \%, p=0.0354$ ) and, in addition, multivariate analysis revealed high dNLR as an independent prognostic factor for OS ( $p=0.0458$ ). (b) There was no significant difference in PFS between the high dNLR and low dNLR groups ( 27.27 vs. $29.9 \%, p=0.2582$ ). (c) The complete response (CR) rate (calculated as stringent CR + CR) was significantly low in the high dNLR group when compared to low NLR group ( 10.8 vs. $26.1 \%, p=0.0148$ ) and, interestingly, in multivariate analysis, high dNLR and advanced age were found to be independent prognostic factors. (d) The safety profile was similar in the 2 groups. Despite the retrospective nature of the study, it revealed dNLR to be a promising prognostic tool for predicting OS in transplant-ineligible patients with newly diagnosed $\mathrm{MM}$ in the era of proteasome inhibitors.

Kim et al. [5] have very recently evaluated the prognostic value of baseline NLR in 273 newly diagnosed MM patients. Multivariate analysis revealed that poor performance status $(\geq 2)$, a high percentage of plasma cells in the bone marrow, a high cytogenetic risk, a high NLR, a low platelet count, elevated C-reactive protein, the use of novel agents (bortezomib, thalidomide, and lenalidomide), and stem cell transplantation were independent and significant prognosticators of OS. The authors designed a new risk classification system termed "The Myeloma Prognostic Index (MPI)” using 3 inflammationbased parameters: baseline high dLNR $(\geq 2.25)$, a low platelet count $(<150.000$ cells $/ \mu \mathrm{L})$, and high $\mathrm{C}$-reactive protein $(\geq 5 \mathrm{mg} / \mathrm{L})$. The MPI score was significantly $(p<$ 0.001 ) associated with OS in both univariate and multivariate analyses regardless of age, renal function, and exposure to novel antimyeloma agents. The median OS was
6.21, 2.43, and 1.01 years for patients with MPI scores of 0 (low), 1 (intermediate), and 2 or 3 (high), respectively. In the subgroup analysis, the MPI scoring system was found to be suitable for effective risk stratification in patients with newly diagnosed MM in terms of treatment type (conventional or novel agents) and age ( $<65$ years transplant-eligible or $\geq 65$ years transplant-ineligible). Furthermore, when the MPI was applied to each ISS risk group (ISS I, II, and III), the patients in each group were divided into 3 additional risk groups (low, intermediate, and high). With this combined approach, all 3 ISS subgroups were clearly stratified. However, this study has major limitations, including its retrospective design, lack of validation in an independent cohort, and the lack of PFS data due to heterogeneous induction regimens as indicated by the authors.

Romano et al. [6] previously described a new prognostic model, "ISS-NLR" which is similar to the MPI. They used ISS together with NLR in nonelderly ( $<65$ years) tranplant-eligible candidates and newly diagnosed $\mathrm{MM}$ patients in a retrospective fashion. This ISS-NLR prognostic model was able to predict PFS and OS in very low, standard, and very high-risk patients, but ISS alone could not. Additionally, these patients were treated upfront with novel antimyeloma agents.

Taken together, pretreatment dNLR might be a potential and applicable biomarker to stratify risk categories and predict survival in newly diagnosed and transplant-ineligible MM patients. Pretreatment dNLR as well as the "MPI" or "ISS-NLR" might be used synergistically either with the ISS or revised ISS. More prospective and large-scale trials are warranted to confirm these promising results.

\section{Disclosure Statement}

There were no conflicts of interest.

\section{References}

$1 \mathrm{Uz} \mathrm{B}$ : Usefulness of baseline neutrophil-tolymphocyte ratio (NLR) in hematological malignancies. Global J Hematol Blood Transfus 2016;3:10-15.

2 Proctor MJ, McMillan DC, Morrison DS, Fletcher CD, Horgan PG, Clarke SJ: A derived neutrophil-to-lymphocyte ratio predicts survival in patients with cancer. Br J Cancer 2012; 107:695- 699 .
3 Troppan K, Deutsch A, Gerger A, Stojakovic T, Beham-Schmid C, Wenzl K, Neumeister P: The derived neutrophil-to-lymphocyte ratio is an independent prognostic factor in patients with diffuse large $\mathrm{B}$-cell lymphoma. $\mathrm{Br}$ J Cancer 2014;110:369-374.

4 Lee GW, Park SW, Go SI, Kim HG, Kim MK, Min CK, et al: The derived neutrophil-tolymphocyte ratio is an independent prognostic factor in transplantation-ineligible patients with multiple myeloma. Acta Haematol 2018, DOI: $10.1159 / 000490488$.
5 Kim DS, Yu ES, Kang KW, Lee SR, Park Y, Sung HJ, et al: Myeloma prognostic index at diagnosis might be a prognostic marker in patients newly diagnosed with multiple myeloma. Korean J Intern Med 2017;32:711-721.

6 Romano A, Parrinello NL, Consoli ML, Marchionni L, Forte S, Conticello C, et al: Neutrophil-to-lymphocyte ratio improves the risk assessment of ISS staging in newly diagnosed MM patients treated upfront with novel agents. Ann Hematol 2015;94:1875-1883. 